\title{
The Performance of the Robo-AO Laser Guide Star Adaptive Optics System at the Kitt Peak $2.1 \mathrm{~m}$ Telescope
}

\author{
Rebecca Jensen-Clem ${ }^{1}$ (1D), Dmitry A. Duev ${ }^{2}$, Reed Riddle ${ }^{2}$ (D), Maïssa Salama ${ }^{3}$ (iD) Christoph Baranec $^{3}$ (D), \\ Nicholas M. Law ${ }^{4}$ (D) S. R. Kulkarni ${ }^{2}$ (D), and A. N. Ramprakash ${ }^{5}$ \\ ${ }^{1}$ Astronomy Department, University of California, Berkeley, CA 94720, USA \\ ${ }^{2}$ Department of Astronomy, California Institute of Technology, 1200 E. California Boulevard, Pasadena, CA 91125, USA \\ ${ }^{3}$ Institute for Astronomy, University of Hawai 'i at Mānoa, Hilo, HI 96720-2700, USA \\ ${ }_{5}^{4}$ Department of Physics and Astronomy, University of North Carolina at Chapel Hill, Chapel Hill, NC 27599-3255, USA \\ ${ }^{5}$ Inter-University Centre for Astronomy \& Astrophysics, Savitribai Phule Pune University Campus, Pune 411 007, India \\ Received 2017 June 20; revised 2017 November 13; accepted 2017 November 18; published 2017 December 21
}

\begin{abstract}
Robo-AO is an autonomous laser guide star adaptive optics (AO) system recently commissioned at the Kitt Peak $2.1 \mathrm{~m}$ telescope. With the ability to observe every clear night, Robo-AO at the $2.1 \mathrm{~m}$ telescope is the first dedicated AO observatory. This paper presents the imaging performance of the AO system in its first 18 months of operations. For a median seeing value of 1". 44, the average Strehl ratio is $4 \%$ in the $i^{\prime}$ band. After post processing, the contrast ratio under sub-arcsecond seeing for a $2 \leqslant i^{\prime} \leqslant 16$ primary star is five and seven magnitudes at radial offsets of $0 . " 5$ and 1." 0 , respectively. The data processing and archiving pipelines run automatically at the end of each night. The first stage of the processing pipeline shifts and adds the rapid frame rate data using techniques optimized for different signal-to-noise ratios. The second "high-contrast" stage of the pipeline is eponymously well suited to finding faint stellar companions. Currently, a range of scientific programs, including the synthetic tracking of near-Earth asteroids, the binarity of stars in young clusters, and weather on solar system planets are being undertaken with Robo-AO.
\end{abstract}

Key words: instrumentation: adaptive optics - instrumentation: detectors - techniques: image processing

\section{Introduction}

Adaptive optics (AO) systems correct wavefront aberrations introduced by the atmosphere and instrumental optics, restoring the angular resolution of a telescope to the diffraction limit. Laser guide stars (LGS) were developed in the 1980s to provide AO systems with bright, locatable wavefront reference sources, thereby bringing fainter astrophysical objects into the purview of AO. Over half of all $8 \mathrm{~m}$ class aperture telescopes are now equipped with an LGS AO system. The primary application of these AO instruments is for high angular resolution studies (deep imaging and spectroscopic) of interesting astronomical objects. As such, minimizing the overhead has not been a major consideration in the overall design of AO systems on large telescopes.

In contrast, Robo-AO is a robotic LGS AO system designed for maximum target throughput. Unlike LGS systems on large telescopes, it is based on an artificial star produced by Rayleigh scattering of a near-UV $(\lambda=355 \mathrm{~nm})$ laser. Robo-AO achieves high target throughput by minimizing overhead times to less than one minute per target. This is accomplished by three key design elements. (1) Each step of the observation sequence is automated, allowing tasks that would be performed sequentially by a human operator to be performed in parallel and with minimal delay by the robotic system. (2) The Rayleigh scattering LGS is invisible to the human eye. As a result, while coordination with the U. S. Air Force Joint Space Operations Center (JSpOC) is still required to prevent illumination of sensitive space assets, no control measures are required by the U. S. Federal Aviation Administration. (3) Robo-AO employs an automated queue scheduler that chooses each new science target based on telescope slew times and approved "lasing" windows provided in advance by JSpOC.
Robo-AO was first commissioned at the Palomar $1.5 \mathrm{~m}$ telescope in 2011, where it completed 19 science runs as a PI instrument from 2012 May through 2015 June. Full details of the Robo-AO hardware and software can be found in Baranec et al. (2013, 2014) and Riddle et al. (2014).

In 2012, the National Optical Astronomy Observatory (NOAO), following the recommendation of the Portfolio Committee, which was chartered by the National Science Foundation (NSF), decided to divest the Kitt Peak (KP) $2.1 \mathrm{~m}$ telescope. In 2015, the Robo-AO team made a bid for the telescope and was selected to operate the telescope for three years. Robo-AO was installed at the $2.1 \mathrm{~m}$ telescope in 2015 November. Since then it has been operating nearly every clear night. As the first dedicated, automated AO facility, Robo-AO at Kitt Peak is well positioned to support the next generation of large-scale survey programs that are focused on stellar and exoplanet astronomy (e.g., CRTS, Pan-STARRS, K2, Gaia, ZTF, TESS, and others), as well as AO follow up of interesting sources. Early science results including Robo-AO KP data can be found in Adams et al. (2017) and Vanderburg et al. (2016a, 2016b).

In this paper, we describe the performance of Robo-AO since commissioning. The paper is organized as follows: in Section 2, we introduce the Robo-AO imaging systems; in Section 3, we provide an overview of our automatic data reduction pipelines; in Section 4, we illustrate the relationships between the weather conditions and the measured seeing; in Section 5, we present the Strehl ratio and the contrast curve statistics as well as the point-spread function morphology; in Section 6, we describe our astrometric solution; in Section 7, we describe our automated data archiving system; finally, in Section 8, we describe the newly installed near-IR camera. 
Table 1

The Specifications of the Robo-AO Optical Detector at Kitt Peak

\begin{tabular}{ll}
\hline \hline Telescope & Kitt Peak 2.1 m telescope \\
Science camera & Andor iXon DU-888 \\
EMCCD detector & E2V CCD201-20 \\
Read-noise (without EM gain) & $47 e^{-}$ \\
EM gain, selectable & $300,200,100,50,25$ \\
Effective read-noise $^{\mathrm{a}}$ & $0.16,0.24,0.48,0.96,1.9 e^{-}$ \\
Full-frame-transfer readout $^{-}$ & 8.6 frames per second \\
Detector format & $1024^{2} 13 \mu \mathrm{m}$ pixels \\
Field of view & $36^{\prime \prime} \times 36^{\prime \prime}$ \\
Pixel scale & $35.1 \mathrm{milli}-\operatorname{arcsec}$ per pixel \\
Observing filters & $g^{\prime}, r^{\prime}, i^{\prime}, z^{\prime}, 1 \mathrm{p} 600$ \\
Standard Exposure Time & $90 \mathrm{~s}$ \\
\hline
\end{tabular}

Note.

${ }^{a}$ The list of effective read-noise values corresponds to the list of EM gain values.

\section{Summary of the Robo-AO Imaging System}

The Robo-AO imaging system includes two optical relays, each using a pair of off-axis parabolic mirrors. The first relay images the telescope pupil onto a 140-actuator Boston Micromachines micro-electro-mechanical-systems (MEMS) deformable mirror used for wavefront correction. A dichroic then reflects the UV light to an $11 \times 11$ lenslet Shack-Hartmann wavefront sensor. The second optical relay includes a fast tip-tilt correcting mirror (so far only used in engineering tests), and an atmospheric dispersion corrector (ADC; here, two rotating prisms ${ }^{6}$ ) located at a reimaged pupil. The output of the second relay is an $f / 41$ beam that is intercepted by a dichroic mirror, which reflects the $\lambda<$ $950 \mathrm{~nm}$ portion of the converging beam to the visible wavelength filter wheel and EMCCD detector (see Table 1). The filter wheel includes $g^{\prime}, r^{\prime}, i^{\prime}$, and $z^{\prime}$ filters, as well as a long-pass "lp600" filter cutting on at $600 \mathrm{~nm}$ and extending beyond the red limit of the EMCCD (see Figure 1 in Baranec et al. 2014). The dichroic transmits the longer wavelength light to the near-infrared (NIR) instrument port (see Section 8).

Robo-AO was originally designed for simultaneous optical and NIR operations, such that deep science integrations could be obtained in one band while the image displacement could be measured in the other and corrected with a fast tip-tilt mirror. In 2017 February, we achieved first light with a science-grade novel infrared array, a brief summary of which appears in Section $8 .^{7}$ In this paper, we consider the imaging performance of Robo-AO using the optical imaging camera only. In lieu of an active tip-tilt correction, the EMCCD is run at a frame rate of $8.6 \mathrm{~Hz}$ to allow for post-facto image registration followed by stacking (see Section 3).

\section{Data Reduction Pipelines}

\subsection{Overview}

Image registration and stacking (see Section 2) is accomplished automatically by the "bright-star" and "faint-star"

\footnotetext{
6 From the commissioning of Robo-AO at Kitt Peak in 2015 November through 2017 February, the right ascension (R.A.) axis of the $2.1 \mathrm{~m}$ telescope suffered from a $\sim 3.7 \mathrm{~Hz}$ jitter (see Section 5.1 and Appendix A) that caused a slight elongation of the stellar point-spread function. As a result, the ADCs were not correctly calibrated until an upgrade to the telescope control system removed the jitter in 2017 February.

7 A detailed analysis of the operation of this camera, its imaging performance, and its incorporation into an active tip-tilt control loop will be reported elsewhere.
}

pipelines, which are optimized for high and low signal-to-noise ratio $(\mathrm{S} / \mathrm{N})$ targets, respectively. The bright-star data are then processed by the "high-contrast" pipeline to maximize the sensitivity to faint companions. These pipelines are described in detail below.

\subsection{Image Registration Pipelines}

All observations are initially processed by the bright-star pipeline. This pipeline centers and windows the time series of images (the "datacube") about an automatically selected guide star. The windowed region is bi-cubically up-sampled and cross correlated with the theoretical PSF to give the center coordinates of the guide star's PSF in each frame. The fullframe, unprocessed images are then calibrated using the nightly darks and dome flats. Finally, the calibrated full frames are aligned using the center coordinates identified by the upsampled, windowed frames, and co-added via the Drizzle algorithm (Fruchter \& Hook 2002). These steps are described in detail in Law et al. (2014).

After an observation has been processed by the bright-star pipeline, the core of the brightest star in the frame is fit by a 2D-Moffat function. If the full width at half maximum (FWHM) of the function fit to the core is $\langle\lambda / \mathrm{D}$, indicating that the stellar centroiding step has failed, then the observation is re-processed by the faint-star pipeline to improve the $S / N$ in the final science image. The cause of the bright-star pipeline failure for faint sources is the $\mathrm{S} / \mathrm{N}$ limitation of the crosscorrelation technique. The typical magnitude cutoff for the bright-star pipeline is $14-15$ in the filter of observation under the median seeing conditions for a $90 \mathrm{~s}$ exposure.

The faint-star pipeline proceeds as follows. The individual frames for a given observation are summed to create a dark and flat corrected "master" reference image. This frame is then high-pass filtered and centered on the guide star. Each raw short exposure frame is then dark and flat corrected, high-pass filtered, and windowed. These individual frames are registered to the master reference frame using the Image Registration for Astronomy package written by Adam Ginsburg. ${ }^{8}$ This python package finds the offset between the individual and reference frames using DFT up-sampling and registers the images with FFT-based sub-pixel image shifts. Figure 1 illustrates the strengths and weaknesses of the bright-star and faint-star pipelines. We note here that the typical centering precision is $\sim 1-5$ mas for the bright-star pipeline and 2-3 times worse for the faint-star pipeline. We see several ways of assessing the accuracy: the formal error of a Moffat/Gaussian fit to a guide star or the FWHM of the core. In the former case, the accuracy is about 10-20 mas, in the latter case 50-100 mas.

These automatic pipelines have reduced thousands of Robo-AO observations since the instrument was commissioned in 2015 November. Figure 2 shows a collage of representative observations.

\subsection{High-contrast Pipeline}

For science programs that aim to identify point sources at small angular separations from known stars, further processing is needed. Our high-contrast pipeline generates a 3".5 frame windowed about the star of interest in the final science frame (we note that the high-contrast pipeline is only applied to those

\footnotetext{
8 https://github.com/keflavich/image_registration
} 


\section{$1 "$}

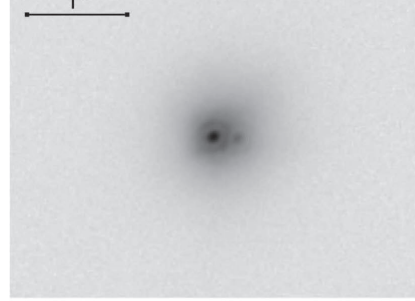

(a) Star: Bright; Pipeline: Bright-star

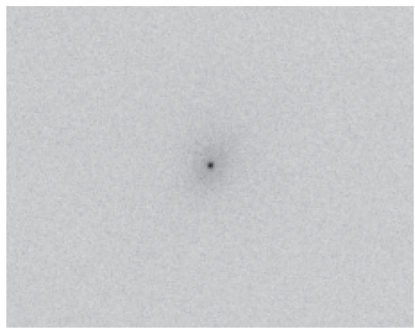

(c) Star: Faint; Pipeline: Bright-star

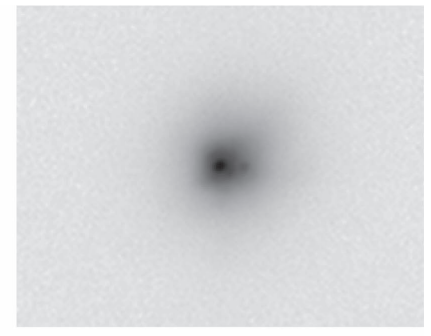

(b) Star: Bright; Pipeline: Faint-star

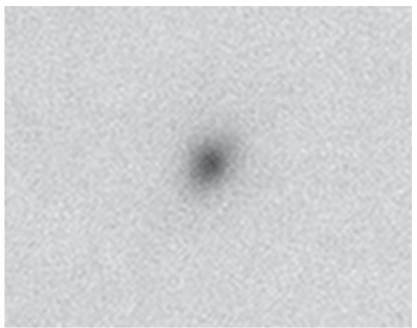

(d) Star: Faint; Pipeline: Faint-star

Figure 1. The bright-star pipeline (a) produces a superior Strehl ratio for the $V=8.84$ double star HIP 55872 compared with (b) the faint-star pipeline. For the $V=15.9$ star 2MASSJ1701+2621, however, the bright-star pipeline (c) fails to correctly center the PSF, leading to an artifact, namely the bright pixel in the center. The faint-star pipeline (d) more accurately registers the individual frames for this observation. All images are displayed with a square root scaling and $\min / \max$ stretch.

stars that were processed by the bright-star pipeline). A highpass filter is applied to the windowed frame to reduce the contribution of the stellar halo (in this pipeline, we used the VIP implementation of the isotropic undecimated wavelet transform with two wavelet scales). To whiten correlated speckle noise at small angular separations from the target star we subtract a synthetic PSF generated by Karhunen-Loève image processing (KLIP). The KLIP algorithm is based on the method of Principal Component Analysis (Soummer et al. 2012). The PSF diversity needed to create this synthetic image is provided by a reference library of Robo-AO observations-a technique called Reference star Differential Imaging (RDI; Lafrenière et al. 2009). We note that the angular differential imaging approach (Marois et al. 2006) is not possible here because the $2.1 \mathrm{~m}$ telescope is an equatorially mounted telescope. Our pipeline uses the Vortex Image Processing (VIP) package developed by Gomez Gonzalez et al. (2017).

The full reference PSF library consists of several thousand 3." 5 square high-pass filtered frames that have been visually vetted to reject fields with more than one point source. The PSF library is updated on a nightly basis to ensure that each object's reduction has the opportunity to include frames from the same night (currently, the library is manually vetted for binaries). Each frame in the full library is cross correlated with the windowed and filtered science frame of interest. The five frames with the highest cross correlation form the sub-library provided to KLIP. We then adopt only the first principal component as our synthetic PSF, as including more components provides no additional improvement to the contrast curve. A clear and desirous extension to the pipeline will be to choose

the number of components automatically for each observation based on $\mathrm{S} / \mathrm{N}$ maximization.

Figure 3 shows an example of a PSF reduced by the standard data pipeline (panel a), then high-pass filtered (panel b), and finally processed with RDI-KLIP (panel $\mathrm{c}$ ). After a science frame has been fully reduced, we use VIP to produce a $5 \sigma$ contrast curve that is properly corrected for small sample statistics and algorithmic throughput losses. The corresponding contrast curves for the three panels are shown in panel (d).

Given that over two hundred new targets are observed during a clear night of Robo-AO observations, the reference library is rapidly expanding and increasingly includes PSFs affected by a very wide range of environmental conditions. Hence, speckle noise in a past observation can be further reduced by a fresh RDI-KLIP reduction if the data is more correlated with later PSFs.

\subsection{Extended Object Pipeline}

The pipelines described in Sections 3.2 and 3.3 are optimized for point-like sources. Robo-AO observations of extended objects, such as solar system planets, are processed by the extended object pipeline. In this pipeline, the individual raw rapid-readout frames are first flat-fielded, dark-subtracted, and sorted by the computed image entropy value, which is used to quantify image sharpness. To limit the computation time, we select a sequence of the 10-20 sharpest frames, and proceed by deconvolving this smaller set (the exact number of frames in the set depends on the object brightness in a particular filterfor instance, we select the 20 sharpest frames for a typical $i^{\prime}$ observation of Jupiter). We are using the deconvolution algorithm implemented in the AIDA software package (Hom et al. 2007). The PSF template used to perform the deconvolution is obtained by (1) selecting the closest in time bright point source observation reduced with the faint-star pipeline $e^{9}$, (2) centering and cropping the frame about this bright point source using the VIP package, (3) padding the cropped frame up to the size of the original full frame, and (4) adding noise to the padded frame from an estimate of the probability density function of the background pixel intensities. An example of an original and deconvolved frame is shown in Figures 4(b) and (c).

The LGS's wavefront samples a cone-shaped volume of the atmosphere where the cone's apex is the height of the laser focus (here $10 \mathrm{~km}$ ) and its base is the telescope's aperture. However, the light from the astronomical source passes through a cylindershaped volume of the atmosphere. The uncorrected wavefront errors to the side of and above the laser's cone induce image distortions which become stronger with the size of the field. This cone effect together with anisoplanatism introduce both plate scale and intensity fluctuations on the image plane. Additionally, the deconvolution procedure may introduce smaller scale artifacts ${ }^{10}$ as the (single) final PSF estimate remains imperfect. For example, it does not account for the cone effect and anisoplanatism so that there are residual artifacts dependant on the position in the frame. To mitigate these effects, we employ an iterative procedure: we shift and add two consecutive frames based on a two-dimensional second-order polynomial. The

\footnotetext{
9 In contrast to that of the bright-star pipeline, the output of the faint-star pipeline is not oversampled and can therefore be directly used in the deconvolution process.

10 ("Smaller" compared to the scale of the artifacts, introduced by the cone effect).
} 


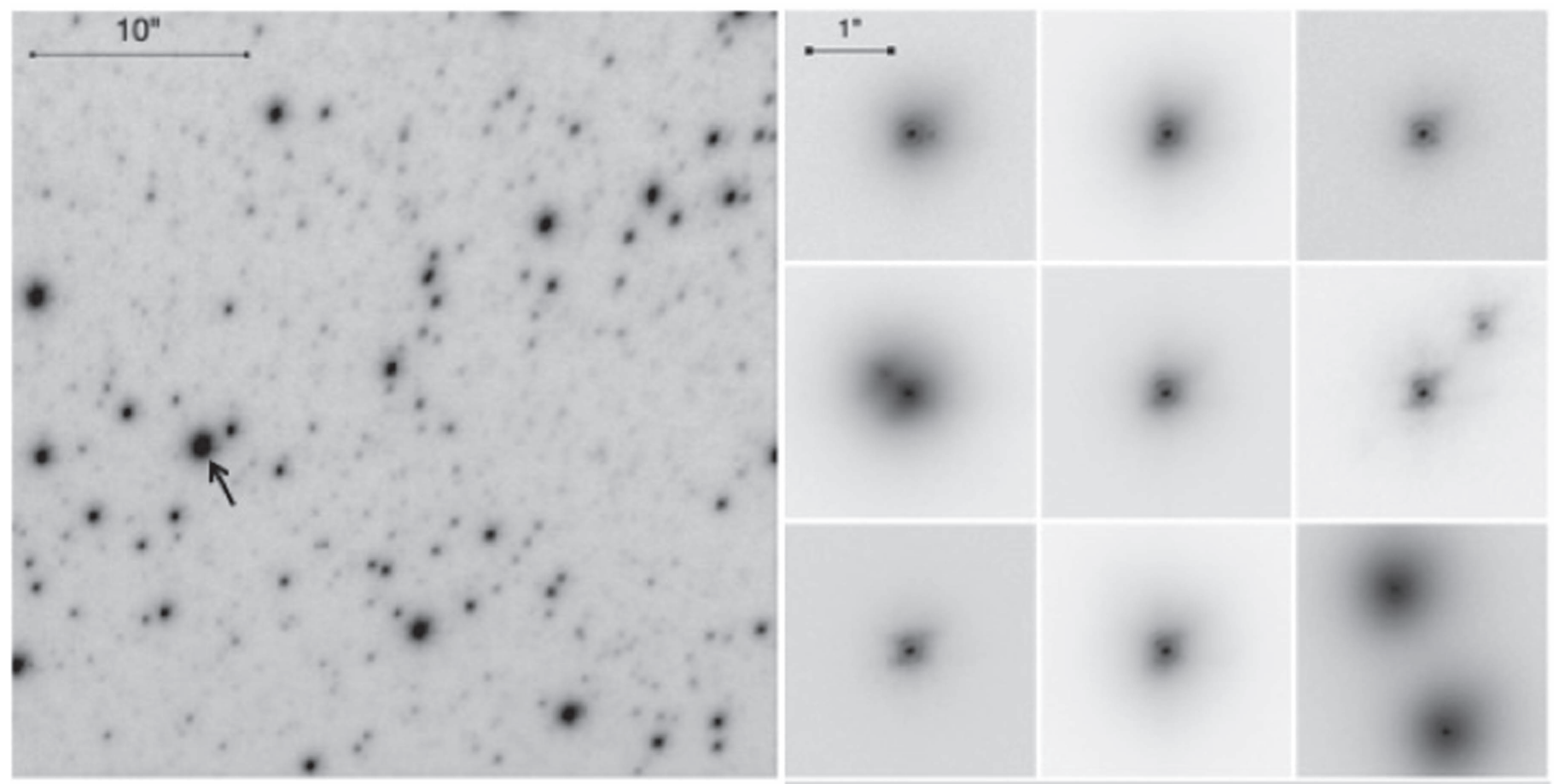

Figure 2. Examples of Robo-AO $i^{\prime}$-band images at the KP $2.1 \mathrm{~m}$ telescope (square root scaling). The left-hand image represents the full $36^{\prime \prime} \times 36^{\prime \prime}$ field of view. In this image of the globular cluster M13, the star used for tip/tilt removal in post processing is indicated by the black arrow. The images on the right are examples of bright single stars and stellar binaries with a range of separations and contrasts $\left(3^{\prime \prime} \times 3^{\prime \prime}\right.$ field of view).

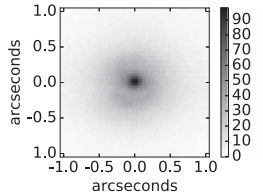

(a) PSF after standard pipeline reduction

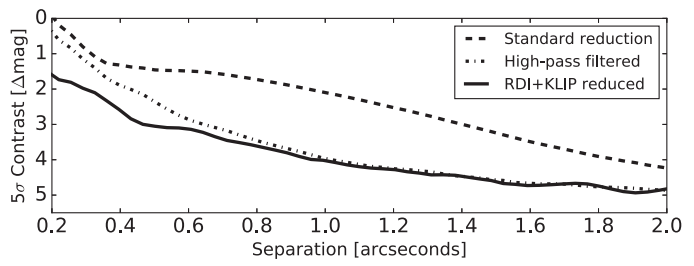

(d) The dashed, dot-dashed, and solid contrast curves correspond to the PSFs shown in (a), (b), and (c), respectively.

Figure 3. An example of the reduction steps in the high-contrast pipeline for a $z^{\prime}$ observation of the star EPIC228859428.

polynomial parameters are iteratively optimized such that the sharpness of the resulting shifted and added image is maximized; see Figure 4(d). Optionally, the resulting stack can be further sharpened using a wavelet-based algorithm described in Ying et al. (2008); see Figure 4(e).

Finally, we note that the deconvolution algorithm implemented in the AIDA software package is effective for densely populated regions like globular clusters, but overshoots for single point sources (or tight binaries). Hence, we only apply this methodology to extended sources in the Robo-AO pipeline.

\section{Site Performance}

\subsection{Site Geography}

Kitt Peak is located 56 miles Southwest of Tucson, Arizona, at an elevation of $6800 \mathrm{ft}$. The $2.1 \mathrm{~m}$ telescope is situated 0.4 miles to the south of the peak's highest point (the location of the Mayall $4 \mathrm{~m}$ telescope). The WIYN 3.5 and $0.9 \mathrm{~m}$ telescopes are respectively $700 \mathrm{ft}$ and $400 \mathrm{ft}$ to the west of the $2.1 \mathrm{~m}$ telescope and at approximately the same elevation. There are no structures at equal or greater elevations to the east of the telescope, and the terrain is relatively flat beyond the Kitt Peak mountain in that direction. The $7730 \mathrm{ft}$ Baboquivari Peak is 12 miles directly south of the telescope.

\subsection{Seeing Measurement}

Before the start of each science observation, a $10 \mathrm{~s}$ observation is taken with the AO correction off. During this period, the wavefront sensor camera acquires a background image. These "seeing" observations are dark and flat calibrated and summed without any registration of the individual exposures. The seeing $\left(\Theta_{s}\right)$, defined as the FWHM of a twodimensional Gaussian function fit to this summed frame, is then extracted. Starting in 2017 January, a $90 \mathrm{~s} \Theta_{s}$ observation was obtained each hour. Specifically, the Robo-AO queue scheduler arranges an observation of a bright $(V<8 \mathrm{mag})$ star within $10^{\circ}$ of the zenith to refocus the telescope and measure $\Theta_{s}$. As of this writing, there is no significant difference between these "long" and "short" $\Theta_{s}$ observations. Here on, we proceed with the assumption that the $10 \mathrm{~s} \Theta_{s}$ measurements are representative of the long-exposure seeing.

We display a histogram of these fiducial $\Theta_{s}$ values in Figure 6. Figure 5 displays $\Theta_{s}$ as a function of the seasons. The $\Theta_{s}$ values measured in a given wavelength are scaled to a 

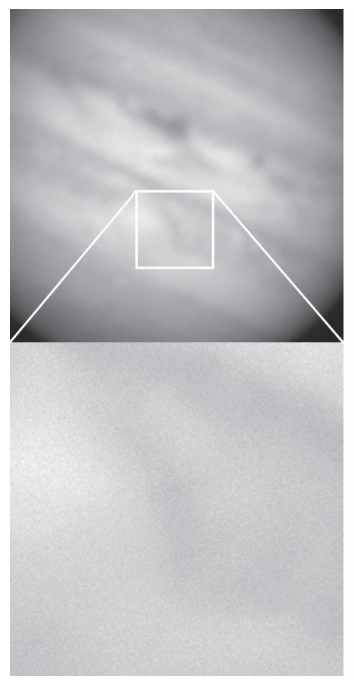

(a) Single seeing-limited frame
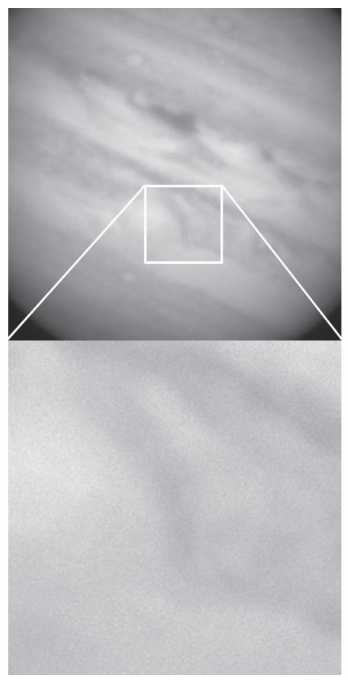

(b) Single frame with $\mathrm{AO}$ on
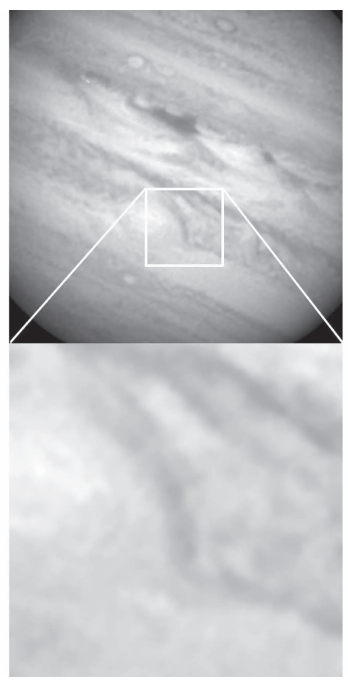

(c) Single frame with AO on, deconvolved

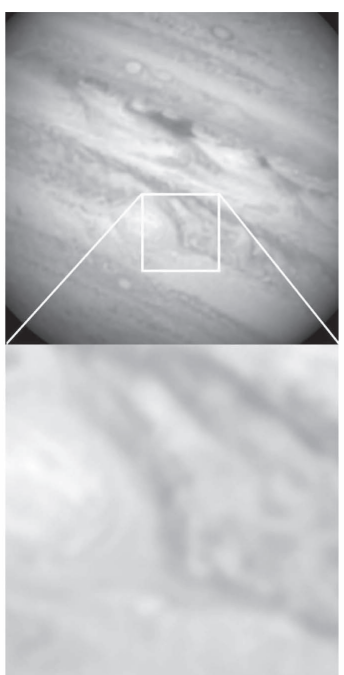

(d) Stack of 20 sharpest deconvolved frames from observation

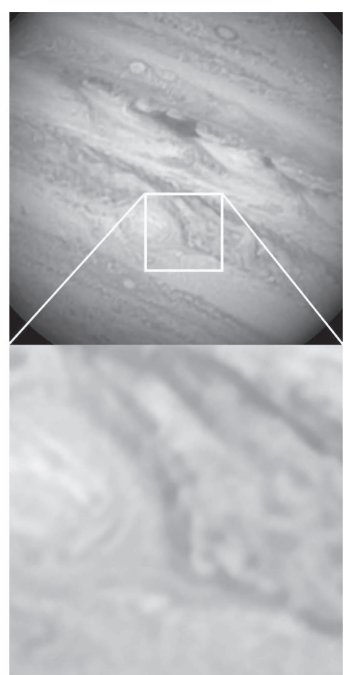

(e) Wavelet-sharpened stack of 20 sharpest deconvolved frames from observation

Figure 4. An example of the reduction steps in the "extended object" pipeline for an $i^{\prime}$ observation of Jupiter taken on 2017 April 7.

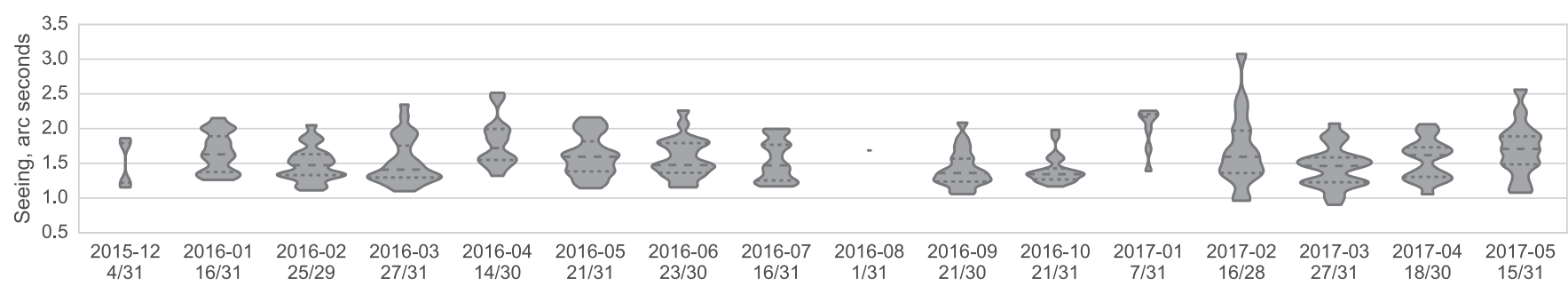

Figure 5. Seasonal fiducial $(\lambda=500 \mathrm{~nm}$; see Section 4.2) seeing measurements. Nightly median values were used to fit a monthly distribution. The fraction of nights with seeing data for each month is shown. The quartile values and the actual measured range are shown.

fiduciary wavelength of $500 \mathrm{~nm}$ by the scaling law

$$
\Theta_{s, 0}(\lambda=500 \mathrm{~nm})=\Theta_{s}(\lambda / 500 \mathrm{~nm})^{1 / 5} .
$$

and are also corrected for zenith distance.

\subsection{Seeing Contributions}

We note that our median seeing of 1 !" 44 differs from the historic median seeing of 0 ". 8 reported by the adjacent WIYN telescope. ${ }^{11}$ One possible explanation for this discrepancy is that the WIYN was built in 1994 with careful attention paid to dome ventilation and telescope thermal inertia. In contrast, the $2.1 \mathrm{~m}$ telescope saw first light in 1964 before such considerations were fully appreciated or designed for. Figure 7 plots the measured Robo-AO seeing values as a function of the difference between the ambient dome temperature and the outside air temperature, where the median temperature difference is $\sim 1^{\circ} \mathrm{C}$. It is clear that larger seeing values are correlated with larger temperature differences (the correlation coefficient is $94 \%$ ). The experience of other observatories indicate that improvements to dome thermalization can significantly improve the measured seeing (e.g., Bauman et al. 2014).

Another possible cause of the comparatively poor seeing at the $2.1 \mathrm{~m}$ telescope is perhaps a more turbulent ground layer. Figure 8 shows a "wind rose," or the frequency of wind speeds

\footnotetext{
${ }^{11}$ http://www.noao.edu/wiyn/aowiyn/
}

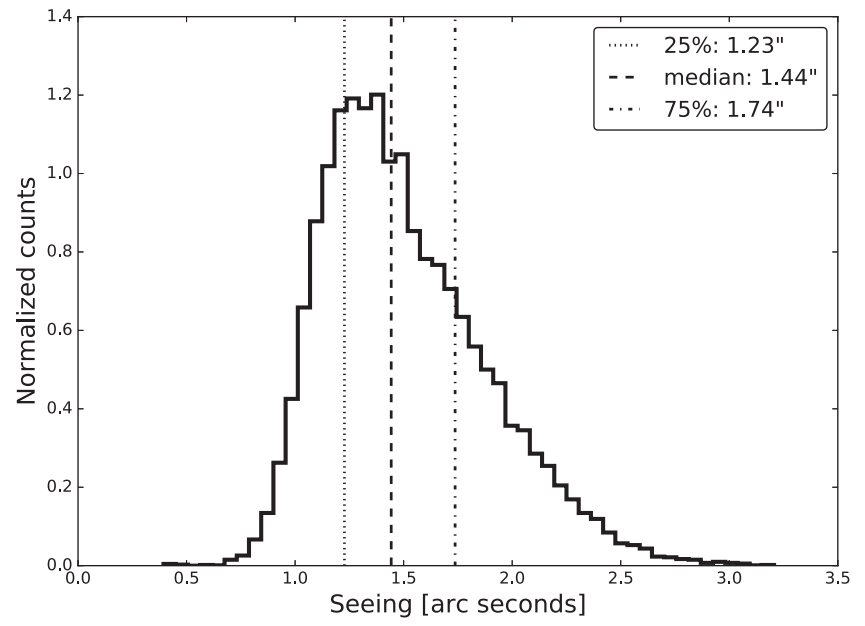

Figure 6. A histogram of the seeing measurements (all referenced to a wavelength $\lambda=500 \mathrm{~nm}$ and Robo-AO's average zenith angle of $20^{\circ}$ ) from 2015 December to 2017 June. The 25th, 50th, and 75th percentile seeing values are indicated by the vertical lines.

originating from different directions, for 2015 December through 2016 June. We find that during this period, the wind most commonly blows from the NNW, or the direction of the higher elevation Mayall $4 \mathrm{~m}$ telescope, and rarely from the SE where the terrain is less mountainous. The highest wind speeds (>40 mph) come from the north while the south has the largest 


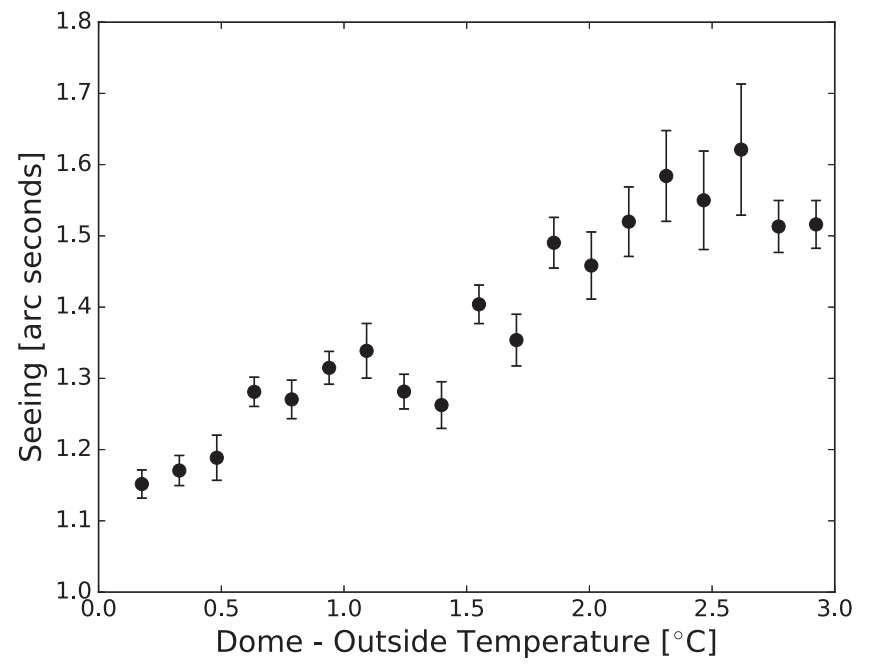

Figure 7. The binned seeing vs. the dome to outside temperature difference from 2017 February to October. The data were binned in units of temperature and the mean taken thereof. The error bars are the standard deviation of the seeing values in a given temperature bin divided by the square root of the number of seeing measurements in the bin.

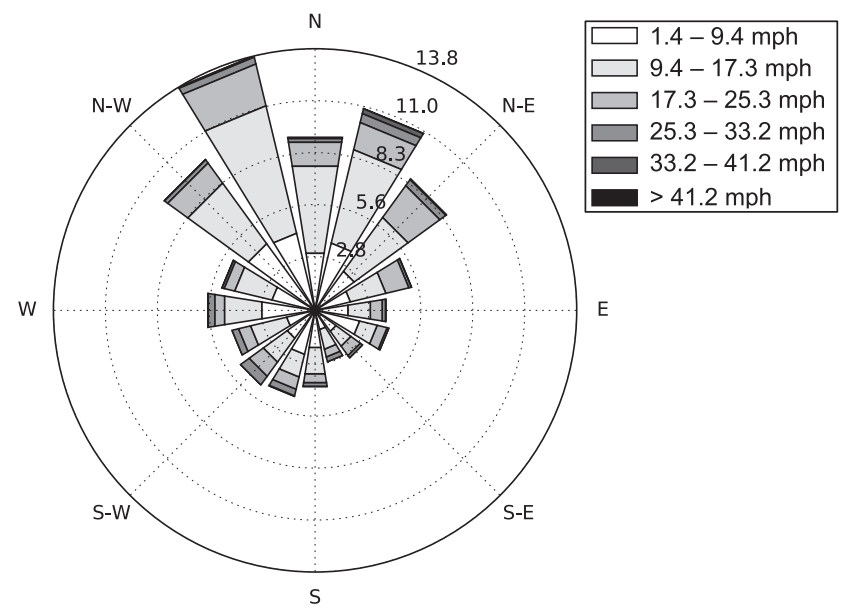

Figure 8. A "wind rose" showing a stacked polar histogram of wind speeds and directions from 2015 December through 2016 June. The wind most frequently blows from the NW, N, and NE, which correspond to the more mountainous region toward the direction of the Mayall $4 \mathrm{~m}$ telescope. These also tend to be the direction of the high wind speeds while slower wind speeds most often come from the south, where the terrain is less mountainous.

fraction of low wind speeds (the wind speeds originating from within $20^{\circ}$ of due south are under $10 \mathrm{mph}, 50 \%$ of the time).

Despite these terrain variations, the seeing is not significantly correlated with the wind direction. The wind speed, however, degrades the seeing by several tenths of an arcsecond for winds over 20 mph. $^{12}$

Figure 9 plots the seeing versus the wind speed, demonstrating that poorer seeing is correlated with higher wind speeds. ${ }^{13}$ Unfortunately the wind monitor stopped working after 2016 June.

Finally, we investigate the contribution of static aberrations to the seeing-limited FWHM. We find the typical size of the static aberrations that are removed by the AO system by comparing our DM flat map with the median of the DM

\footnotetext{
12 The dome closes when the wind speed exceeds $40 \mathrm{mph}$.

13 The mean binned seeing measurements in Figure 9 are larger than the median of all Robo-AO KP seeing measurements (Figure 6) due to binning effects and the difference between the mean and median of the asymmetric distribution of seeing measurements.
}

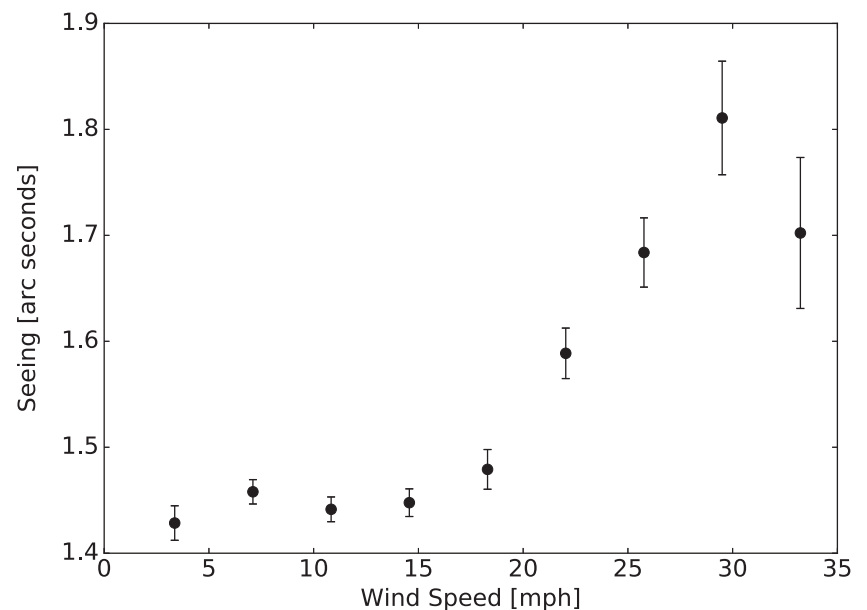

Figure 9. The binned seeing vs. the wind speed for 2015 December through 2016 June. The data were binned in units of $\mathrm{mph}$ and the mean taken thereof. The error bars are the standard deviation of the seeing values in a given wind speed bin divided by the square root of the number of seeing measurements in the bin. For wind speeds over $20 \mathrm{mph}$, the seeing is degraded by up to 0.13 .

actuator positions from a night of $\sim 1^{\prime \prime}$ seeing. The rms of the difference between the DM flat map and this median DM position is $277 \mathrm{~nm}$. Because the open loop rms wavefront error is $>1 \mu \mathrm{m}$ (Noll 1976), we conclude that static aberrations do not significantly inflate our seeing measurements.

\section{AO Performance}

\subsection{Strehl Ratio}

The goal of an AO system is to bring the observed PSF closer to its theoretical diffraction-limited shape. Hence, an important measure of the AO system's performance is the ratio between the peak intensity of an observed PSF and that of the telescope's theoretical PSF-the Strehl ratio. As the AO performance improves, the Strehl ratio increases.

We calculate the Strehl ratio by (1) generating a monochromatic diffraction-limited PSF by Fourier transforming an oversampled image of the pupil, (2) combining several monochromatic PSFs to create a PSF representative of the desired bandpass, (3) re-sampling the polychromatic PSF to match our 0." $0175 /$ pixel plate scale of the up-sampled "drizzled" frames, (4) obtaining the "Strehl factor," or the ratio of the peak intensity (i.e., the maximum of the centered, drizzled, and summed image) to the sum of the intensity in a $3^{\prime \prime}$ square box, and (5) calculating the Strehl ratio by repeating step 4 for the observed image and dividing by the Strehl factor. These steps are described in detail in Salama et al. (2016).

Regular observation began in 2015 December. After this time, we noticed that the achieved Strehl ratios were noticeably worse than those that were achieved (for similar seeing values) at the Palomar $1.5 \mathrm{~m}$ telescope. A number of investigations were undertaken to determine possible causes for this degradation. Eventually, we determined that the Telescope Control System (TCS) was the main contributing factor. In Appendix A, we discuss the problem in detail. We solved this issue by upgrading the TCS (completed in 2017 February). Below, and for the rest of the paper, we discuss the instrument performance since the TCS upgrade. 


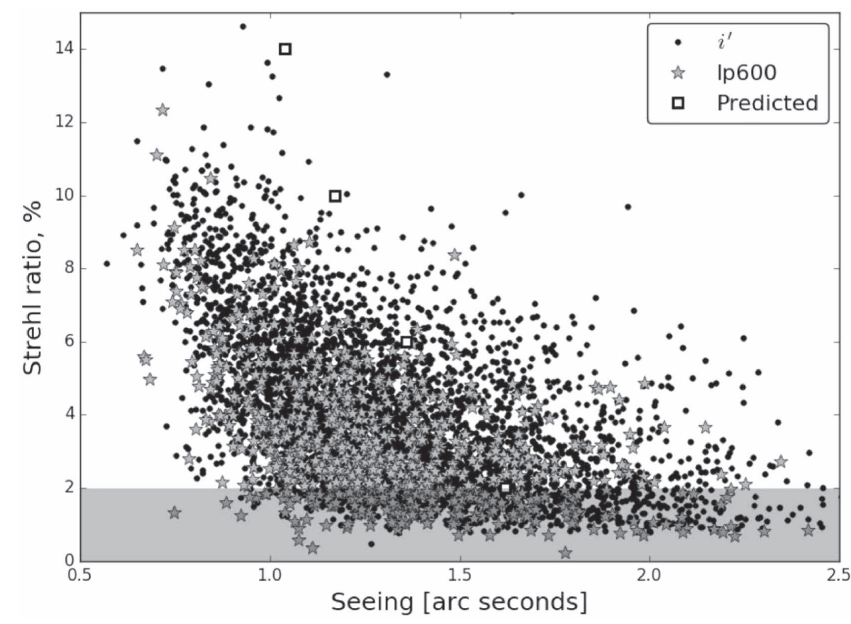

Figure 10. The Strehl ratio vs. the measured seeing values for 2017 February 21 through June 4 in the $i^{\prime}$ and lp600 filters. The predicted values from Table 2 are overplotted. The shaded region $(0 \% \leqslant \mathrm{Strehl} \leqslant 2 \%)$ indicates the region where our Strehl ratio calculations become inaccurate.

Figure 10 plots the Strehl ratio versus the measured seeing for the $i^{\prime}$ and lp600 filters. It is clear that the delivered Strehl ratio drops off quickly as the seeing increases-while RoboAO achieves $>10 \%$ Strehl ratio when the seeing is $<1$ ". 0 , a 0 "' 25 seeing increase halves the Strehl ratio.

In Table 2, we present a detailed error budget under different seeing conditions. This error budget was originally developed by R. Dekany (private communication), and was validated against the on-sky performance of laser AO systems on the Keck and Hale $5 \mathrm{~m}$ telescopes, as well as Robo-AO at the Palomar $1.5 \mathrm{~m}$ telescope (Baranec et al. 2012). As we lacked turbulence profile(s) for the $2.1 \mathrm{~m}$ telescope site, we adopt a mean $C_{n}^{2}(h)$ profile measured using a MASS-DIMM atmospheric turbulence monitor collected over a year's baseline at Palomar and scaled to the seeing at Kitt Peak. The error budget's high-order terms are added in quadrature. The singleaxis tip or tilt errors are estimated to be dominated by bandwidth error for magnitudes greater than 13 (for fainter objects, measurement error dominates as we were unable to precisely determine the centroid of the star due to the lower number of photons per exposure). As noted in Section 2, we did not use the built-in tip-tilt facility but instead resorted to shift and add. We approximate the error resulting from this approach as follows. We assume a standard $-3 \mathrm{db}$ rejection frequency matching the frame rate of the science camera to approximate bandwidth error. The tip-tilt errors are then converted to an equivalent wavefront error and summed in quadrature with the high-order errors. Other high-order and tiptilt errors include chromatic, scintillation, aliasing, calibration, and digitization errors. The Strehl ratios in Table 2 are calculated using the Maréchal approximation (Maréchal 1947). The FWHM are calculated from PSF models assuming the residual diffraction-limited, residual seeing, and scattered light halos are proportional to the phase variance of the residual errors. Sheehy et al. (2006) have shown that the accuracy of these models are a few percent for Strehl ratios as low as $4 \%$.

Figure 10 demonstrates Robo-AO's ability to approach the predicted Strehl ratio of $14 \%$ in sub-arcsecond seeing conditions. There is significant variation, however, in the measured Strehl ratios for a given seeing value. This is due to variations in the nature of the turbulence. Figure 11, for example, shows two observations of the globular cluster M3. Both observations were taken when the seeing was $\sim 1$ !" 8 , but the Strehl ratio associated with one of the observations was $5.4 \%$ while the other was $3.6 \%$. Because the Strehl ratio is a physically motivated quantity (the fraction of the total starlight inside the diffraction-limited core of the PSF), it is a more accurate predictor of the image image quality than the seeing measurement (an approximate measure of the width of the uncorrected PSF). Hence, the Strehl ratio, rather than the seeing value, is a more reliable predictor for image quality in Robo-AO observations.

\subsection{PSF Morphology}

Figure 12 shows a representative Robo-AO PSF corresponding to the $V=10$ star HIP 56051. The observation was taken in the $i^{\prime}$ band with a total exposure time of $90 \mathrm{~s}$. The seeing at the time of the observation was 0.'94, and the Strehl ratio of the final PSF is $10.17 \%$.

The effect of the AO system is to re-arrange the starlight from the equivalent area seeing-limited PSF (dashed curve) to the sharper, observed PSF plotted by the black points. The AOcorrected PSF includes two components: a sharp core and a broader halo, each separately fit by Moffat functions (the light and dark gray curves, respectively). The FWHM of the Moffat function fit to the core is 0 ." $1 \pm 0$." 01 . This value is consistent with the diffraction limit of $1.028 \lambda / \mathrm{D}=0$ !. 08 .

\subsection{Contrast Curves}

Section 3 described the high-contrast pipeline, which produces $5 \sigma$ contrast curves from the high-pass filtered, RDIPCA reduced science frames. Figure 13 plots the median and best $10 \%$ contrast curves for $i^{\prime}$ and 1 p600 filter science frames, with typical exposure times of $90 \mathrm{~s}$. Under sub-arcsecond seeing (the best $10 \%$ of cases), the contrast ratio for a $2 \leqslant i^{\prime} \leqslant 16$ primary star is five and seven magnitudes at 0 ". 5 and 1 !" 0 , respectively.

\section{Astrometric Solution}

In order to register Robo-AO images to sky coordinates and correct for field distortions, we must obtain the instrument's astrometric solution. This process is crucial for precision astrometry applications such as solar system object tracking (for example near-Earth asteroids) and the measurement of multiple star systems' position angles and separations. To this end, we use nightly observations of densely populated globular clusters including M3, M5, and M13 to establish and update the astrometric solution. Each night's globular cluster observations are flat-fielded, dark-subtracted, and reduced by the bright-star pipeline.

The first step in obtaining the astrometric solution is to locate all point sources in a globular cluster image, a task made easier by image deconvolution. We deconvolve our reduced images using the method described in Section 3.4. Next, we detect individual sources in the deconvolved image and extract their pixel positions using the SExtractor software package. We proceed if a sufficient number of sources has been detected.

To identify the sky positions of the extracted sources, we take advantage of the milli-arcsecond positional information 
Table 2

The Robo-AO Error Budget

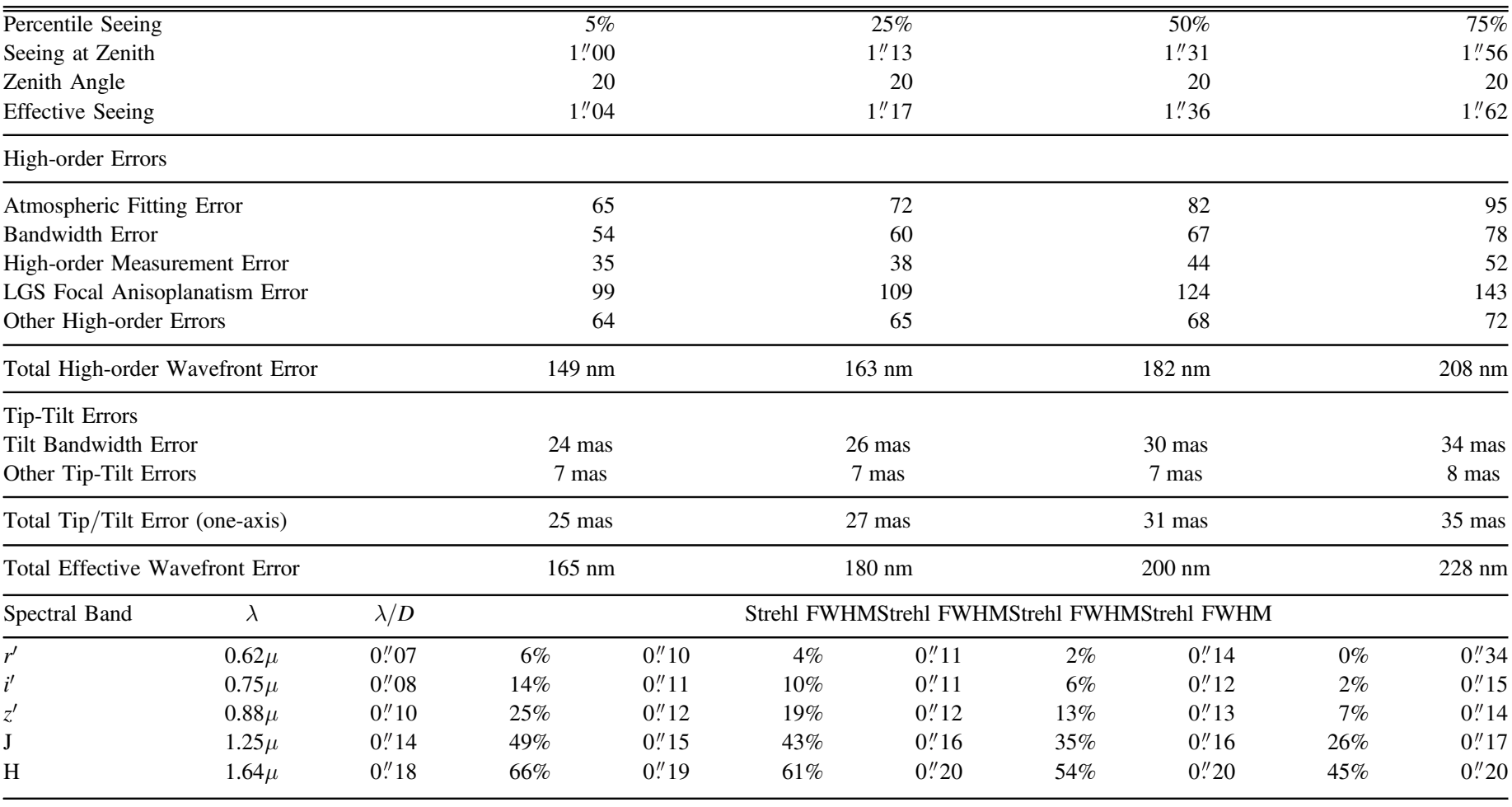

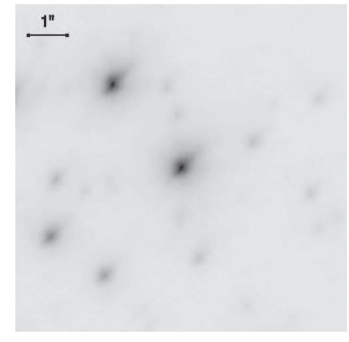

(a) An observation of M3 with a Strehl Ratio of $5.4 \%$.

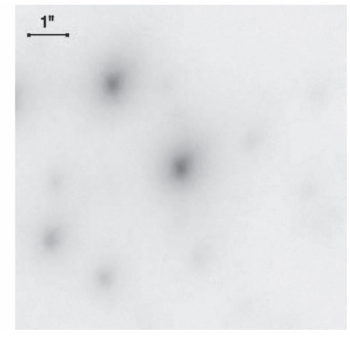

(b) An observation of M3 with a Strehl Ratio of $3.6 \%$.

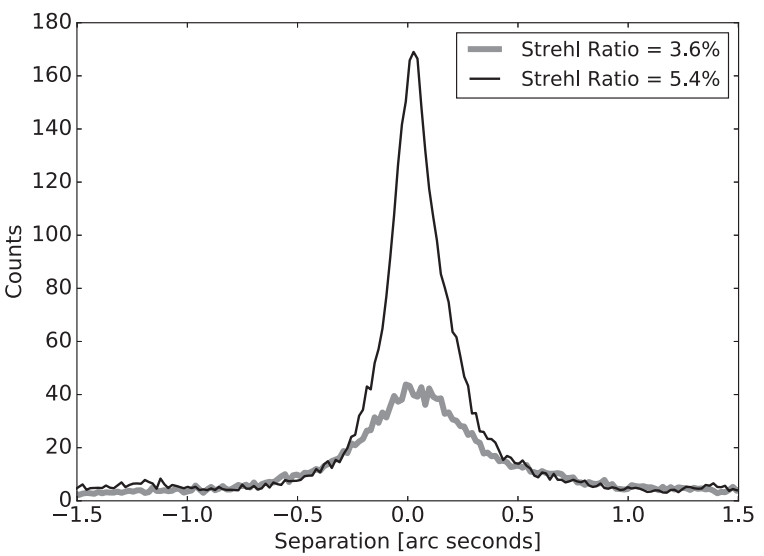

(c) A comparison of the PSFs of the central star pictured in (a) and (b).

Figure 11. Two observations of the globular cluster M3 have different Strehl ratios, but their nearest measured seeing values were both 1 !. 8 .

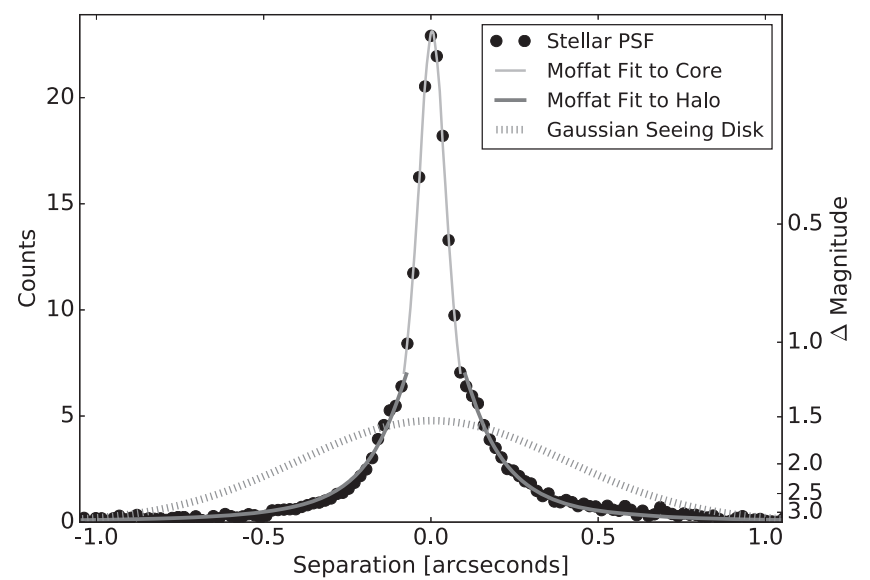

Figure 12. A one-dimensional cut through the PSF of HIP 56051 is plotted with two Moffat functions fit to the PSF core and halo, respectively. The dashed curve is a Gaussian distribution with an FWHM corresponding to the seeing measurement and an area equal to the observed PSF's area.

provided by the Gaia Mission Data Release 1 (DR1) catalog (Gaia Collaboration et al. 2016). We query the DR1 catalog within $100^{\prime \prime}$ of the center of the globular cluster image (recorded by the $2.1 \mathrm{~m}$ TCS in the "TELRA" and "TELDEC" FITS header keywords). We retain only those stars that are brighter than a Gaia magnitude of 16 (typically, 70-100 stars are successfully cross-matched to the Gaia catalog).

Next, we cross-match the sources extracted from our deconvolved image with the Gaia sources. This is accomplished by first generating a coarse a priori astrometric solution to project the Gaia star positions onto the detector plane. We 


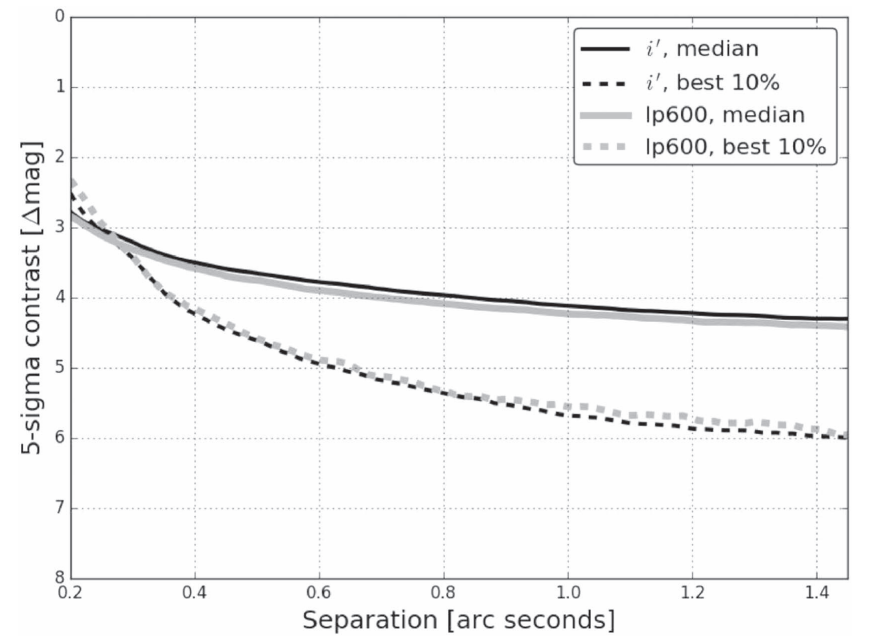

Figure 13. The contrast as a function of distance from the central star for the $i^{\prime}$ and lp600 filters (with 157 and 53 images, each with an individual exposure time of $90 \mathrm{~s}$, analyzed for the $i^{\prime}$ and 1 p600 filters respectively). The solid and dashed lines show the median and best $10 \%$ contrast curves for each filter. The data has been processed with the RDI-PCA pipeline described in Section 3.

then generate a synthetic image by convolving these projected positions with the PSF estimated by AIDA. A synthetic "detected" image, generated using the detected pixel coordinates of stars, accordingly centered and padded, is then correlated with the synthetic reference image to provide the offset that is used to cross-match the stars.

The pixel coordinates of a star $i$ in the detector plane $\left(x_{i}, y_{i}\right)$ are related to its sky position (R.A. $i$, decl.i) via the expressions derived in K. Mierle \& D. W. Hogg (2007, in preparation) ${ }^{14}$ and given in Appendix B here. The estimated field distortion map is shown in Figure 14. We note that the Robo-AO system contains a double optical relay with off-axis parabolas (OAPs), which are known to introduce quadratic distortion. The full astrometric solution (a linear mapping and a second-order distortion) is given in Appendix B and is also available on the Robo-AO archive. The typical difference between an object's Gaia sky coordinates and those derived using the estimated detector plane-to-sky mapping is 5 mas.

\section{Data Archive}

We have developed a fully automated data processing and archiving system. ${ }^{15}$ The data reduction chain for an observing night proceeds as follows. At the end of each night, the visual camera data are compressed and transferred to the network storage. Next, the darks and dome flats taken at the beginning of each night are combined into master calibration files and applied to the observations. The bright-star pipeline is then run on each observation followed by the computation of the Strehl ratio of the resulting image. The high-contrast pipeline also produces highpass filtered, PSF-subtracted images and contrast curves for each of these processed images (see Section 3). If the drizzled image produced by the bright-star pipeline does not pass a quality check (i.e., if a two-component Moffat fit to the PSF has an anomalously narrow core or wide halo) then the faint-star pipeline re-reduces the rapid readout data. Additionally, the "archiver" processes the nightly seeing data and generates summary plots of the seeing measurements, Strehl ratios, and

\footnotetext{
14 http://astrometry.net/svn/trunk/documents/papers/wcs-tutorial/wcs.tex

15 https://github.com/dmitryduev/archiver
}

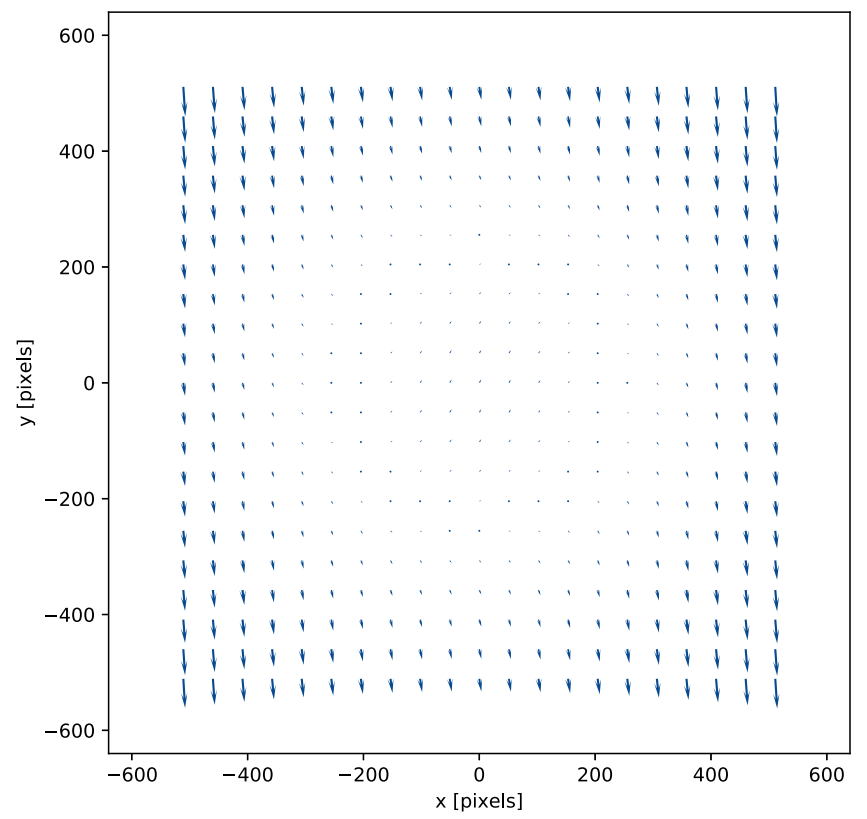

Figure 14. The estimated field distortion map in the detector space. The arrows represent the displacement of each grid point and are $2 \times$ magnified for better visual perception.

contrast curves. Completing the full reduction chain for a typical night's worth of data takes a few hours.

The "house-keeping" system uses the dask. distributed library for distributed computation ${ }^{16}$ to manage the processing queue. The processing results together with ancillary information on individual observations and system performance are stored in a MongoDB ${ }^{17}$ NoSQL database. For interactive data access, we developed a web-based interface ${ }^{18}$ powered by the $\mathrm{Flask}^{19}$ back-end. It allows the user to access previews of the processing results together with auxiliary data (e.g., external VO images of a field), nightly summary, and system performance information. The web application serves as the general interface to the database providing a sophisticated query interface.

\section{Near-infrared Camera}

In 2016 November, we installed an NIR camera for use with Robo-AO. The detector is a $320 \times 256$ pixel Mark 13 Selex ES Avalanche Photodiode for High-speed Infrared Array (SAPHIRA) with a $\lambda=2.5 \mu \mathrm{m}$ cutoff and sub-electron readnoise. It uses a ME-911 readout integrated circuit as described in Atkinson et al. (2016) and Baker et al. (2016). The singleboard PB1 "PizzaBox" readout electronics were developed at the University of Hawaii Institute for Astronomy. We use 32 readout channels, each capable of a 2 Mpixel s$^{-1}$ sampling rate, for a maximum full-frame read rate of $\sim 800 \mathrm{~Hz}$. Compared with a similar engineering NIR camera tested at the Palomar $1.5 \mathrm{~m}$ telescope in 2014 (Baranec et al. 2015), our current NIR camera includes a science-grade detector and faster readout electronics.

The NIR camera attaches to the Robo-AO $f / 41$ infrared camera port that accesses $\lambda>950 \mathrm{~nm}$ after transmission through a dichroic. The camera has an internal cold short-pass

\footnotetext{
16 https://github.com/dask/distributed

17 http://www.mongodb.com

18 http://roboao.caltech.edu/archive

19 https://github.com/pallets/flask
} 
Figure 15. A $5.5 \mathrm{~s}$ image of the M-dwarf binary system GJ1116 AB taken in $H$-band with the near-infrared camera (linear stretch).

$(\lambda<1.85 \mu \mathrm{m})$ filter and an external warm filter wheel with $J$, $H$, clear, and blocking filters. The camera has a plate scale of 0 " 064 per pixel and field of view of 16 ". $5 \times 20$ ". 6 .

We achieved first light on sky in 2017 February during the final testing of the upgraded TCS. Initially, we used a $1 \mathrm{Mpixel} /$ second sampling rate (a full-frame read rate of $390 \mathrm{~Hz}$ ) with detector resets every 300 reads. To create a reduced image, we first assembled difference frames between 39 consecutive reads, totaling $\sim 0.1 \mathrm{~s}$ of integration time, short enough to effectively freeze stellar image displacement. We subtracted a frame median to approximate removing the background. We then synthesized a long-exposure image by registering each corrected frame on the brightest target in the field. Figure 15 shows an example image of a binary star observed in $H$-band.

Currently, data acquisition and reduction is performed manually. In the coming months, we will optimize the detector readout routines for maximum sensitivity to faint objects (including dithering for background removal), integrate the operation of the camera into the robotic queue, and modify our existing data reduction pipeline to handle the NIR data. We intend to investigate automating active tip-tilt correction by using either the visible or infrared camera as a tip-tilt camera, as previously demonstrated at Palomar.

\section{Conclusion}

Robo-AO at the Kitt Peak $2.1 \mathrm{~m}$ telescope is the first dedicated AO observatory. Robo-AO has the capacity to undertake LGS AO surveys of large samples. For instance, a 1000 star survey with exposure times of $60 \mathrm{~s}$ per target can be completed on the timescale of a week.

Science programs designed to exploit Robo-AO's unique capabilities are underway. These programs include stellar multiplicity in open clusters, minor planet binarity, major planet weather variability, extragalactic object morphology, sub-stellar companions to nearby young stars, M-star multiplicity, and the influence of stellar companions on asteroseismology. By the end of 2017, on-going upgrades to the $2.1 \mathrm{~m}$ telescope and dome will enable fully automated operations without the need for a human safety monitor.

The Robo-AO team thanks NSF and NOAO for making the Kitt Peak $2.1 \mathrm{~m}$ telescope available. We would like to thank Richard R. Treffers (Starman Systems, LLC) for his contribution to the upgrade of the $2.1 \mathrm{~m}$ Telescope Control System (TCS). We thank the observatory staff at Kitt Peak for their efforts to assist Robo-AO KP operations. Robo-AO KP is a partnership between the California Institute of Technology, the University of Hawai ' $\mathrm{i}$, the University of North Carolina at Chapel Hill, the Inter-University Centre for Astronomy and Astrophysics (IUCAA) at Pune, India, and the National Central University, Taiwan. The Murty family feels very happy to have added a small value to this important project. Robo-AO KP is also supported by grants from the John Templeton Foundation and the Mt. Cuba Astronomical Foundation. The Robo-AO instrument was developed with support from the National Science Foundation under grants AST-0906060, AST0960343, and AST-1207891, IUCAA, the Mt. Cuba Astronomical Foundation, and by a gift from Samuel Oschin. These data are based on observations at Kitt Peak National Observatory, National Optical Astronomy Observatory (NOAO Prop. ID: 15B-3001), which is operated by the Association of Universities for Research in Astronomy (AURA) under cooperative agreement with the National Science Foundation. C.B. acknowledges support from the Alfred P. Sloan Foundation.

Facility: KPNO:2.1m (Robo-AO).

Software: Image Registration for Astronomy, VIP (Gomez Gonzalez et al. 2017), AIDA (Hom et al. 2007), SExtractor (Bertin \& Arnouts 1996), MongoDB, Flask.

\section{Appendix A \\ Telescope Jitter}

After moving Robo-AO from the Palomar $1.5 \mathrm{~m}$ telescope to the Kitt Peak $2.1 \mathrm{~m}$ telescope, the median Strehl ratio across all wavelengths was initially reduced from $5.8 \%$ to $3.2 \%$. The source of this degradation was a $\sim 3.7 \mathrm{~Hz}$ vibration in the R.A. axis. Because Robo-AO mitigates tip/tilt by post-facto shift and add rather than a real-time loop, and because its frame rate is typically only $8.6 \mathrm{~Hz}$, the targets were smeared in the R.A. direction.

Figures 16(a) and (b) show the superimposed power spectral densities of the mean subtracted R.A. centroid positions of each target observed at Kitt Peak and Palomar, respectively. The peak at $\sim 3.7 \mathrm{~Hz}$ is clear in the Kitt Peak data, but is not present at Palomar. The R.A.-axis smearing for a single test observation is demonstrated in Figure 17.

The jitter was mitigated by two changes to the system. First, the KPNO staff noticed a ticking sound corresponding to each rotation of the telescope drive worm gear, which was solved by lubrication. This step reduced the height of, but did not eliminate, the PSD peak. Second, we took a test observation in which only sidereal tracking was enabled, and all fine computer guiding was turned off. The peak was absent in this test observation, leading us to conclude that the TCS was giving erroneous commands that "kicked" the telescope's position. The TCS upgrade in the winter of 2017 eliminated the remaining R.A. jitter (Figure 18). Figure 19 shows a comparison of the Strehl ratios versus the seeing before and after the TCS upgrade.

\section{Appendix B Mapping Image Coordinates to Sky Coordinates}

The pixel coordinates of a star $i$ in the detector plane $\left(x_{i}, y_{i}\right)$ are related to its sky position (R.A. ${ }_{i}$, decl.i) via the following 


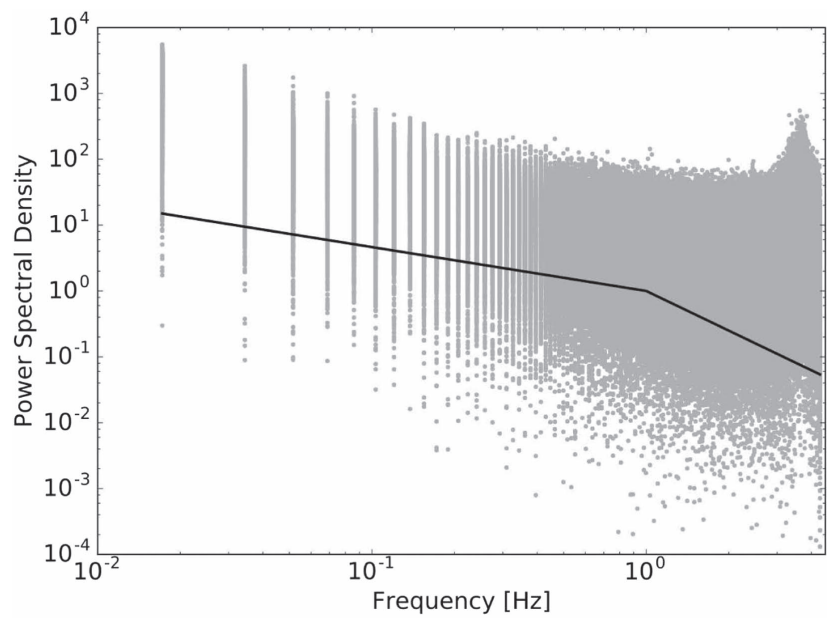

(a) Kitt Peak mean subtracted RA centroids

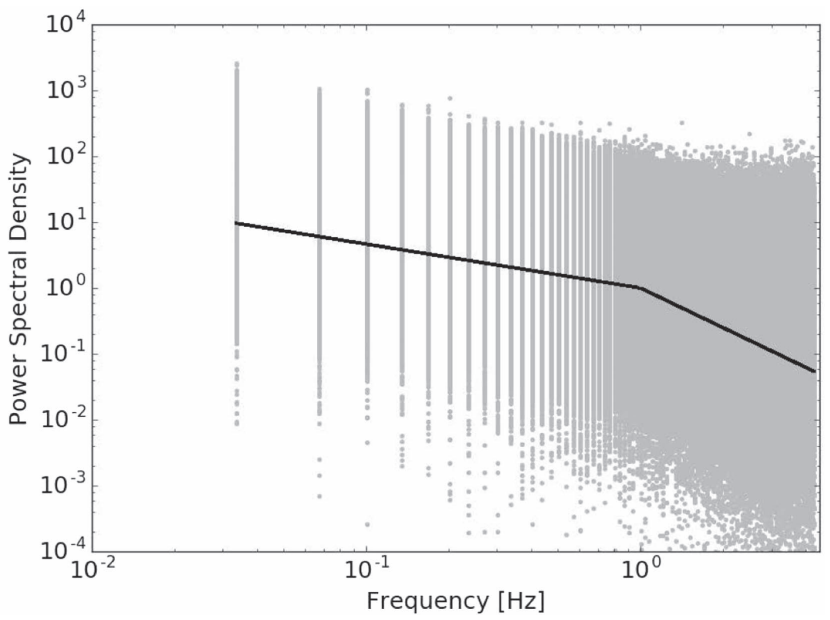

(b) Palomar mean subtracted RA centroids

Figure 16. The power spectral densities of the mean subtracted R.A. target positions for each sub-exposure at Kitt Peak (a) and Palomar (b). The peak at $\sim 3.7$ Hz is present at Kitt Peak, but not at Palomar. The solid black lines show the theoretical power-law dependencies of the tilt: $f^{-2 / 3}$ at low frequencies, and $f^{-2}$ for $1-10 \mathrm{~Hz}$ (Hardy 1998)

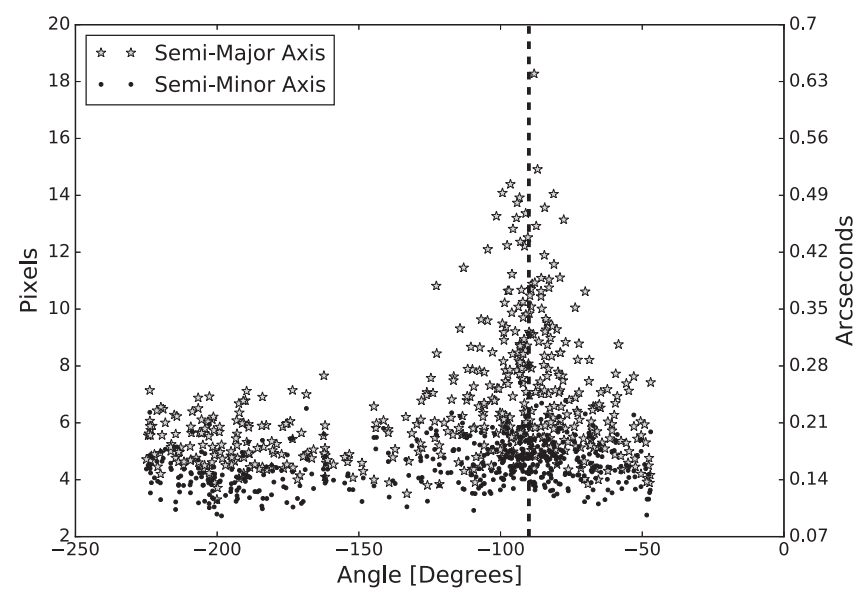

Figure 17. For a test observation, the standard deviation along the semimajor and semiminor axes of two-dimensional Gaussian fits to each $0.116 \mathrm{~s}$ subexposure are plotted vs. the rotation angle of the Gaussian. Here, $-90^{\circ}$ (dashed black line) indicates that the semimajor axis lies along the R.A.-axis. Clearly, the PSF is elongated along the R.A.-axis.

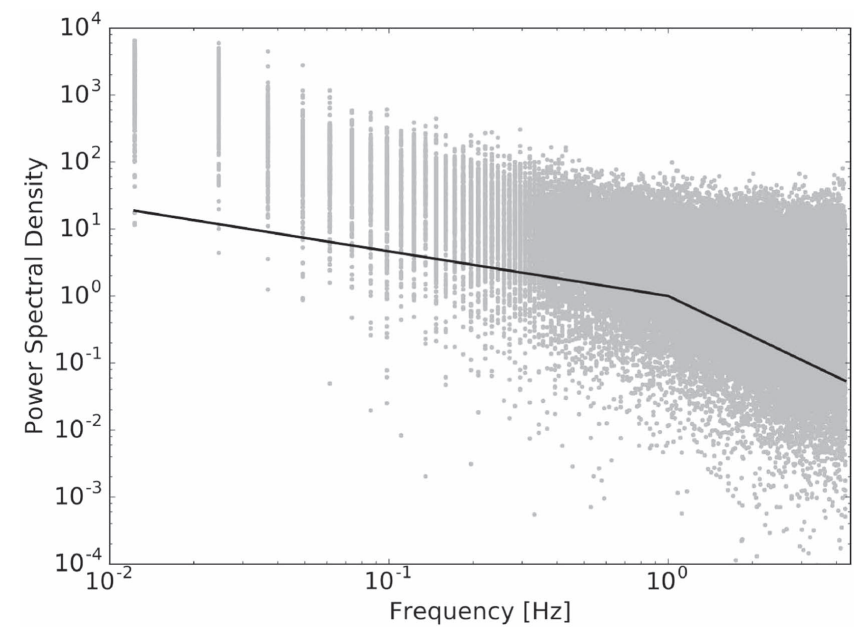

Figure 18. The power spectral densities of the mean subtracted R.A. target positions for the Kitt Peak sub-exposures since the telescope control upgrade (2017 February 22 through March 8). The peak that was present in Figure 16(a) is eliminated.

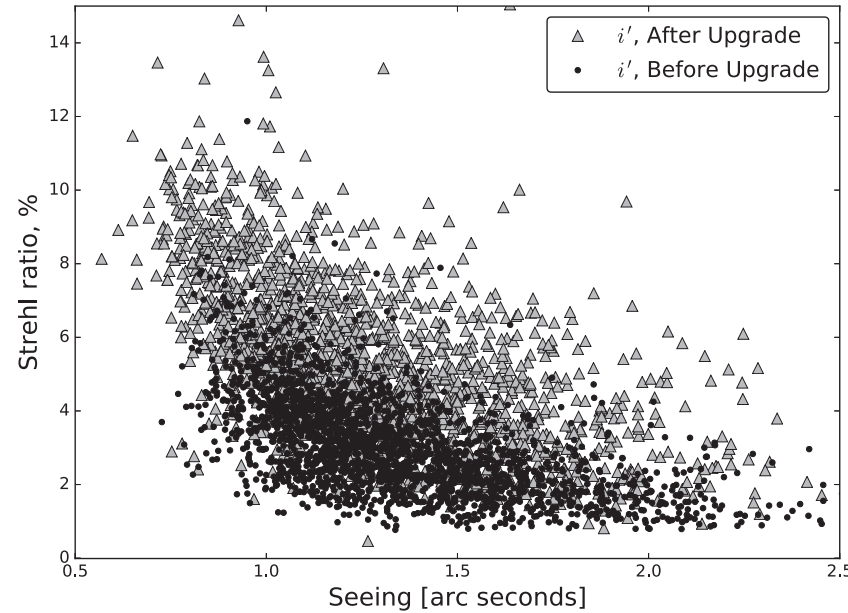

Figure 19. Strehl ratios of the observations taken in $i^{\prime}$ as a function of the seeing scaled to $500 \mathrm{~nm}$ before (2015 December through 2017 February 22; black points) and after (2017 February 22 through June 4; gray stars) the enhancements. Note the significant improvement for seeing under $\approx 1.1$ arcseconds.

set of equations (K. Mierle \& D. W. Hogg 2007, in preparation). ${ }^{20} \mathrm{We}$ are using the tangent projection approach given that our field of view is quite small. The "native" tangent plane position of the $\operatorname{star}\left(u_{i}, v_{i}\right)$ is given by:

$$
\left[\begin{array}{c}
u_{i} \\
v_{i}
\end{array}\right]=\mathrm{M} \cdot\left[\begin{array}{l}
\Delta x_{i}+f\left(\Delta x_{i}, \Delta y_{i}\right) \\
\Delta y_{i}+g\left(\Delta x_{i}, \Delta y_{i}\right)
\end{array}\right]
$$

where

$$
\left[\begin{array}{l}
\Delta x_{i} \\
\Delta y_{i}
\end{array}\right]=\left[\begin{array}{l}
x_{i}-x_{\tan } \\
y_{i}-y_{\tan }
\end{array}\right]
$$

$f(x, y)$ and $g(x, y)$ are polynomial functions of order $N_{f}$ and $N_{g}$ that describe distortion on the tangent plane, $\left(x_{\tan }, y_{\tan }\right)$ is the location of the tangent point in detector pixel coordinates, and a 2-by-2 matrix $M$ (measured in deg pix $^{-1}$ ) gives the linear relationship between native and pixel coordinates.

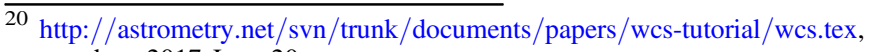
accessed on 2017 June 20.
} 
The polynomials $f(x, y)$ and $g(x, y)$ have coefficients $a_{p q}$ and $b_{p q}$

$$
\begin{aligned}
& f(x, y)=\sum_{p=0}^{N_{f}} \sum_{\substack{q=(2-p) \\
q \geqslant 0}}^{\left(N_{f}-p\right)} a_{p q} x^{p} y^{q} \\
& g(x, y)=\sum_{p=0}^{N_{g}} \sum_{\substack{\left(N_{g}-p\right) \\
q=(2-p) \\
q \geqslant 0}} b_{p q} x^{p} y^{q},
\end{aligned}
$$

where the limits on the $q$ summation ensure that there are no zeroth order or linear terms and no terms above order $N_{f}$ or $N_{g}$ (that is, no terms with $p+q>N_{a}$ or $p+q>N_{b}$ ). As noted in Section 6, the Robo-AO system contains a double optical relay with OAPs, which introduce quadratic distortion. We therefore set $N_{f}=2$ and $N_{g}=2$. Equation (4) is then expanded as follows:

$$
\begin{aligned}
& f(x, y)=a_{02} y^{2}+a_{11} x y+a_{20} x^{2} \\
& g(x, y)=b_{02} y^{2}+b_{11} x y+b_{20} x^{2},
\end{aligned}
$$

The vector position in the tangent plane $\boldsymbol{R}_{i}$ is just a scaling back to the natural units of the native coordinates (expressed as a vector), which are relative to the tangent point, plus the vector pointing to the position of the tangent point $\hat{t}$ :

$$
\boldsymbol{R}_{i}=\hat{\boldsymbol{t}}+\frac{\pi}{180}\left\{u_{i} \hat{\boldsymbol{u}}+v_{i} \hat{\boldsymbol{v}}\right\}
$$

where the unit vectors are defined in an ICRS realization, and $\hat{\boldsymbol{t}}$ is given by:

$$
\begin{aligned}
\hat{\boldsymbol{t}}= & \cos \left(\mathrm{R}^{\mathrm{A}} \mathrm{A}_{\cdot \tan }\right) \cos \left(\mathrm{decl}_{\cdot \tan }\right) \hat{\boldsymbol{i}} \\
& +\sin \left(\mathrm{R}^{\mathrm{A} \cdot \tan }\right) \cos \left(\mathrm{decl}_{\tan }\right) \hat{\boldsymbol{j}} \\
& +\sin \left(\text { decl }_{\tan }\right) \hat{\boldsymbol{k}} .
\end{aligned}
$$

To get back onto the celestial sphere, the vector position in the tangent plane must be re-scaled back to unit length:

$$
\hat{\boldsymbol{r}}_{i}=\frac{\boldsymbol{R}_{i}}{\left|\boldsymbol{R}_{i}\right|} .
$$

Finally, the celestial position (R.A.i, decl. $_{i}$ ) of the star is given by inverse trigonometric functions of components of the unit vector $\hat{\boldsymbol{r}}_{i}$ :

$$
\begin{aligned}
\text { R.A. }_{\cdot i} & =\arctan \left(\hat{\boldsymbol{r}}_{i} \cdot \hat{\boldsymbol{j}}, \hat{\boldsymbol{r}}_{i} \cdot \hat{\boldsymbol{i}}\right) \\
\operatorname{Decl}_{\cdot i} & =\arcsin \left(\hat{\boldsymbol{r}}_{i} \cdot \hat{\boldsymbol{k}}\right),
\end{aligned}
$$

where the function $\arctan (y, x)$ takes the inverse tangent of the ratio $y / x$ but properly accounts for the quadrant.

Given the pixel and sky coordinates of a set of stars detected on the image, we run a least-squares estimation of the transformation parameters. We do a few reiterations of the estimation process, first refining the initial coarse parameter values, and subsequently discarding the stars that show large residuals. We use the statistical bootstrap method to estimate the confidence intervals for the parameters. The current best estimates of the transformation parameters with $1 \sigma$-errors are as follows. The components of the matrix $M$ :

$$
\begin{aligned}
& M_{11}=-9.924338 \times 10^{-6} \pm 6.6 \times 10^{-11} \\
& M_{12}=9.1952 \times 10^{-8} \pm 8.5 \times 10^{-11} \\
& M_{21}=-1.940 \times 10^{-8} \pm 2.1 \times 10^{-10} \\
& M_{22}=9.719203 \times 10^{-6} \pm 9.3 \times 10^{-11}
\end{aligned}
$$

which corresponds to a rotational angle of 0.112 east from the north, and mean pixel scales in the $x$ and $y$ directions of $0 . \prime 0357277$ and $00^{\prime \prime} 0349884$, respectively. The pixel scales, in turn, yield an image size of 36 ". 5137 by 35 ". 7582 . The location of the tangent point in detector pixel coordinates is $(-1.70 \pm 0.04,-2.95 \pm 0.01)$. Finally, the coefficients in Equation (5) are:

$$
\begin{aligned}
& a_{02}=-7.23 \times 10^{-7} \pm 1.02 \times 10^{-7} \\
& a_{11}=-3.61 \times 10^{-7} \pm 2.01 \times 10^{-7} \\
& a_{20}=2.70 \times 10^{-7} \pm 1.14 \times 10^{-7} \\
& b_{02}=-5.5066 \times 10^{-5} \pm 2.2 \times 10^{-8} \\
& b_{11}=3.00 \times 10^{-7} \pm 1.54 \times 10^{-7} \\
& b_{20}=-5.3787 \times 10^{-7} \pm 3.2 \times 10^{-8} .
\end{aligned}
$$

\section{ORCID iDs}

Rebecca Jensen-Clem 주 https://orcid.org/0000-00030054-2953

Reed Riddle (1) https://orcid.org/0000-0002-0387-370X

Maïssa Salama (10) https://orcid.org/0000-0002-5082-6332

Christoph Baranec (1) https://orcid.org/0000-0002-1917-9157

Nicholas M. Law (1) https://orcid.org/0000-0001-9380-6457

S. R. Kulkarni (i) https://orcid.org/0000-0001-5390-8563

\section{References}

Adams, E. R., Jackson, B., Endl, M., et al. 2017, AJ, 153, 82

Atkinson, D. E., Hall, D. N. B., Baker, I. M., et al. 2016, Proc. SPIE, 9915, $99150 \mathrm{~N}$

Baker, I., Maxey, C., Hipwood, L., \& Barnes, K. 2016, Proc. SPIE, 9915, 991505

Baranec, C., Atkinson, D., Riddle, R., et al. 2015, ApJ, 809, 70

Baranec, C., Riddle, R., Law, N. M., et al. 2013, JVE, 72, e50021

Baranec, C., Riddle, R., Law, N. M., et al. 2014, ApJL, 790, L8

Baranec, C., Riddle, R., Ramaprakash, A. N., et al. 2012, Proc. SPIE, 8447, 844704

Bauman, S. E., Benedict, T., Baril, M., et al. 2014, Proc. SPIE, 9149, 91491K

Bertin, E., \& Arnouts, S. 1996, A\&AS, 117, 393

Fruchter, A. S., \& Hook, R. N. 2002, PASP, 114, 144

Gaia Collaboration, Brown, A. G. A., Vallenari, A., et al. 2016, A\&A, 595, A2

Gomez Gonzalez, C. A., Wertz, O., Absil, O., et al. 2017, AJ, 154, 7

Hardy, J. W. 1998, Adaptive Optics for Astronomical Telescopes (Oxford: Oxford Univ. Press)

Hom, E. F. Y., Marchis, F., Lee, T. K., et al. 2007, JOSAA, 24, 1580

Lafrenière, D., Marois, C., Doyon, R., \& Barman, T. 2009, ApJL, 694, L148

Law, N. M., Baranec, C., \& Riddle, R. L. 2014, Proc. SPIE, 9148, 91480A

Maréchal, A. 1947, Rev. Opt. Theor. Instrum., 26, 257

Marois, C., Lafrenière, D., Doyon, R., Macintosh, B., \& Nadeau, D. 2006, ApJ, 641,556

Noll, R. J. 1976, JOSA, 66, 207

Riddle, R. L., Hogstrom, K., Papadopoulos, A., Baranec, C., \& Law, N. M. 2014, Proc. SPIE, 9152, 91521E

Salama, M., Baranec, C., Jensen-Clem, R., et al. 2016, Proc. SPIE, 9909, 99091A

Sheehy, C. D., McCrady, N., \& Graham, J. R. 2006, ApJ, 647, 1517

Soummer, R., Pueyo, L., \& Larkin, J. 2012, ApJL, 755, L28

Vanderburg, A., Becker, J. C., Kristiansen, M. H., et al. 2016a, ApJL, 827, L10

Vanderburg, A., Bieryla, A., Duev, D. A., et al. 2016b, ApJL, 829, L9

Ying, L., Ming, N. T., \& Keat, L. B. 2008, in International Conference on Computer Science and Software Engineering (New York: IEEE), 1053, https://doi.org/10.1109/CSSE.2008.1631 